\title{
External diathermy treatment of proliferative diabetic retinopathy with vitreous haemorrhage
}

\author{
ABDEL-LATIF SIAM \\ From Ain Shams University, Cairo, Egypt
}

SUMMARY Sixty-six cases of advanced proliferative diabetic retinopathy were treated by external diathermy. The eyes had extensive fibrovascular proliferations growing into the vitreous. All had some blood, recent and old, in the vitreous. The vitreous was extremely degenerated and retracted. All the patients had inadequate diabetic control. More than half were hypertensive, with reasonable medical control, while a few had some degree of renal failure, with lower limb oedema. None of the cases were suitable for photocoagulation. Thirty-two control eyes were followed up together with the treated eyes for one to eight years. Of 53 eyes of patients with maturity onset diabetes 28 improved, 12 remained unchanged, and 13 deteriorated. Only two eyes developed neovascular glaucoma, while eight of 13 eyes of patients with juvenile diabetes improved. Of the control eyes, one regressed spontaneously, six remained unchanged, and 25 deteriorated. Of these 25 eyes eight developed traction detachment, 10 suffered relentless attacks of vitreous haemorrhage, and seven developed neovascular glaucoma. External diathermy appears to be a logical approach to cases which could not be treated by either photocoagulation or vitrectomy.

At present the major lines of treatment for proliferative diabetic retinopathy (PDR) are photocoagulation therapy and vitrectomy procedures. Buckling techniques have also been applied to cases of traction detachment, due to diabetic retinopathy.' The efficacy of photocoagulation becomes progressively less with the more advanced stages of PDR owing to fibrovascular proliferation and traction detachment. This line of therapy is out of the question if considerable amounts of blood are present, particularly in the vitreous gel. Nor can it be applied to cases with marked corneal or lens opacities.

There remain only two possibilities for retinal coagulation, namely cryotherapy and external diathermy. No results are available of a controlled study on the effects of external diathermy for cases of PDR in which photocoagulation treatment is impossible and vitrectomy procedures are not indicated. Wessing and Bockenhoff ${ }^{2}$ reported on the effect of treatment by intrascleral diathermy and have observed that haemorrhages into the vitreous are usually quickly absorbed. We made a preliminary report on the beneficial effects of external diathermy on the course of PDR with vitreous haemorrhage. ${ }^{3}$

Correspondence to Professor A-L Siam, 3 Shagaret El-Dorr Street, Zamalek, Cairo, Egypt.
Cryothermy has also been tried for such cases, ${ }^{4}$ but there is no definitive study on this line of treatment.

The natural history of such cases is unpredictable. A minority start to regress and continue to dry up and quieten down both as regards the vitreous blood and the fibrovascular elements; even the traction detachment regresses. Fibrous arcs, with limited detachment, persist in such cases. The patient retains some walking vision and occasionally may enjoy aided reading ability. ${ }^{3}$

However, when the course is regressive, whether spontaneously or following external diathermy treatment, more glial formations have been observed, often with traction detachment. The last was associated with extensive vascular sheathing and attenuation, with apparently extensive ischaemic areas marked by fibrovascular formations at their limits. This condition was more marked in the untreated cases and was followed either by neovascular glaucoma or by optic atrophy (see 'Results' below).

However, most of the untreated cases $(25$ out of 32 , i.e. $78 \%$ ) continued to progress to more fibrovascular proliferations, and then developed either extensive traction detachment or a state of old, persistent vitreous haemorrhage.

Many of these eyes developed neovascular glaucoma and cataract, with an unsightly painful eye 
( 7 out of 32 , i.e. $22 \%$ ). Of the late developments rhegmatogenous retinal detachment and retinoschisis appear to be the result, with more fibrosis and more vitreous degeneration and retraction, resulting in both static and dynamic vitreous traction.

Vitrectomy techniques are a great advance in dealing with some cases of old-standing vitreous haemorrhage and of traction retinal detachment. But such elaborate surgical approaches, including the separation, cutting, and circumcision of epiretinal membranes, ${ }^{5}$ are not indicated early in the course of progressive diabetic retinopathy with recurrent attacks of vitreous haemorrhage and active neovascular activity.

\section{Material and methods}

\section{PATIENTS}

Sixty-six eyes of PDR were treated with external diathermy coagulations. Together with 32 control eyes, mostly the other eye of treated patients, they were followed up for one to eight years. The duration of diabetes ranged from five to 35 years. Thirteen cases had type I diabetes, and their ages ranged from 20 to 35. Fifty-three cases were of maturity onset or type II diabetes, with ages of 45 to 70 years. The control of diabetes was poor and irregular in all cases. Forty-three cases were receiving antihypertensive drugs, with reasonable control of their hypertension.

Their visual acuity ranged from $1 / 60$ to $6 / 24$.

Anterior segment examination did not show any iris new vessels on slit-lamp examination. The vitreous was found to be retracted and contracted, with vitreovascular and vitreoglial adhesions in all cases where it could be satisfactorily examined.

Fifty-six cases had varying amounts of vitreous blood, but the fundus could be visualised to allow reliable evaluation of the fundus changes. Ten cases presented with massive, recent, bright red vitreous blood. Six cases had partial retinal detachment involving more or less than one quadrant of the retina.

Eight cases had lower limb oedema, with marked albuminuria and lid puffiness.

The 66 treated cases shared the following features. All had extensive fibrovascular proliferations arising from the disc and from the retinal surface. The areas of surface proliferations were several disc diameters, while the disc vessels were already pulled into the vitreous for varying distances. More than half of the cases had extrapapillary retinovitreal proliferations. All had vitreous blood in quantities which ranged from an amount that allowed reasonable visualisation of fundus details (56 cases) to massive vitreous blood that did not allow any view of the fundus (10 cases). The last group of cases had either been under our care before the attack of vitreous haemorrhage, and therefore had a record of the stage of their PDR, or were studied only by ultrasonography, providing data on the vitreous echos, vitreous membranes, and the position of the retina. The electroretinogram (ERG) was also done in these cases, in some with a bright flash.

Twelve cases had been treated by photocoagulation, xenon or argon laser, before the attack of vitreous haemorrhage.

Thirty-two control cases had the same features as the treated cases and were randomly chosen. They received aspirin tablets only in addition to their antidiabetic treatment, with special insistence on as much attention as possible to diet and diabetic control and the avoidance of straining efforts.

Fasting blood sugar values ranged from 120 to $350 \mathrm{mg} / \mathrm{dl}(6 \cdot 7-19 \cdot 4 \mathrm{mmol} / \mathrm{l})$. Six cases had acetone in their urine when first seen, but only two of these women had repeated acetonuria in the follow-up. Four of them were in the treatment group.

\section{METHOD}

Seventy to $801 \mathrm{~mm}$ diameter applications of 50-60 mA for 2 seconds were enough to produce a yellowish white reaction in all cases, with reasonable fundus visibility. The coagulations were placed over the upper and lower temporal quadrants, above and

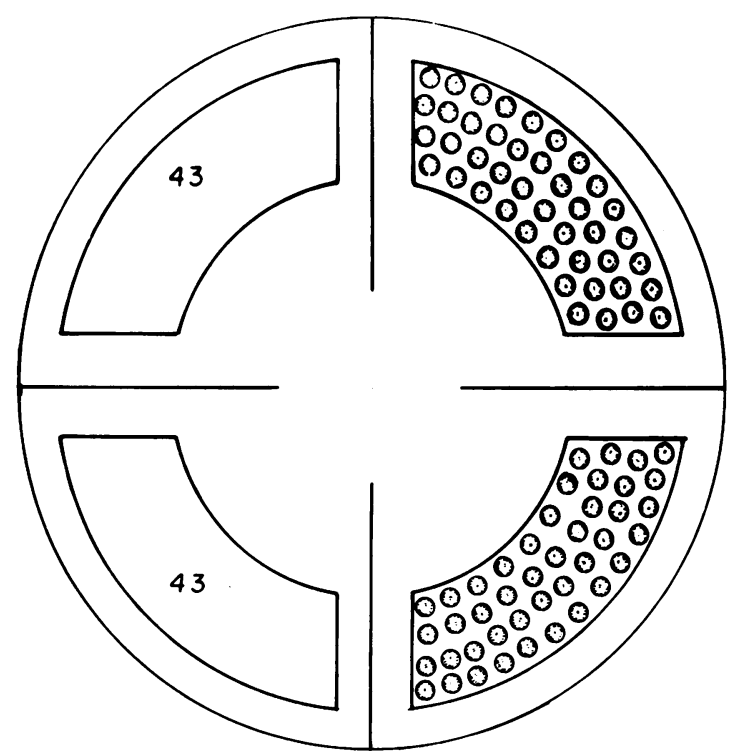

Fig. 1 Shows the pattern of diathermy applications each 50-60 mA for 2 seconds. Forty-three $1 \mathrm{~mm}$ diameter applications placed over upper and lower temporal quadrants, above and below the lateral rectus muscle; parallel rows, each row staggered in relation to the next. 
below the lateral rectus muscle, sparing the temporal long posterior ciliary artery. They were made in rows parallel to the equator, each row being staggered in relation to the next (Fig. 1).

In nine cases the operation was repeated over the nasal quadrants, again sparing the nasal long posterior ciliary artery. Twelve cases had had previous panretinal photocoagulation (PRP) (Table 3).

At first the Hamblin ophthalmic diathermy unit was used, but later the unipolar radiofrequency Mira diathermy unit was used at a setting of 5-6 intensity for 1-2 seconds. When visibility was reasonable, control of the intensity of coagulations was possible. Overreactions were always avoided; overshrinkage of the sclera was not allowed, especially over thin scleral areas. The raised ocular tension was controlled by repeated paracentesis; in all cases the globe was left with firm tension. I made sure that the retinal vessels were at least pulsating at the end of the coagulation procedure. In the cases where the fundus was not visible the tension was kept below $35 \mathrm{mmHg}$.

\section{Results}

Of 53 cases (Table 1) of maturity onset diabetes 28 $(53 \%)$ improved, with visual results attained between $1 / 60$ and $6 / 24$. Twelve eyes $(22 \%)$ remained almost unchanged, while 13 eyes $(25 \%)$ steadily deteriorated to more fibrosis and traction detachment. It was observed, however, that only two of these suffered repeated attacks of vitreous haemorrhage, and only two eyes developed neovascular glaucoma (less than $4 \%$ ). Five ended in traction detachment (less than 10\%) and four showed optic atrophy and vascular sclerosis.

Of the cases treated again (nine eyes) six improved and three deteriorated, while of the 12 eyes that had previous PRP 10 improved and two got worse (Table 2).

Of 13 eyes of juvenile (type I) diabetes treated, eight $(61.5 \%)$ improved, the retinopathy quietening almost completely, with few white retinovitreal and papillovitreal white bands. Five cases deteriorated, two suffering further attacks of vitreous haemorrhage, two traction detachment, and one ending in neovascular glaucoma.

\section{COMPLICATIONS}

Ocular hypertension followed the diathermy applications in all cases and, unless controlled with repeated paracentesis, could lead to acute ischaemic optic atrophy. Choroiditis and vitritis occurred in four cases, while reactionary retinal detachment was observed in three. This complication took several days to clear up; only one case took more than one week to disappear.
Table 1 Numbers and types of diabetic cases treated with external diathermy and results of treatment.

\begin{tabular}{llll}
\hline & \multicolumn{2}{l}{ Treated cases } & \\
\cline { 2 - 4 } & Total & Type II & Type I \\
& 66 & 53 & 13 \\
\hline Improved & & 28 & 8 \\
Unchanged & 12 & 0 \\
Deteriorated & 13 & 5 \\
VH & 2 & 2 \\
TD & 5 & 2 \\
NG & 2 & 1 \\
OA & 4 & 0 \\
\hline
\end{tabular}

$\mathrm{VH}=$ vitreous haemorrhage $. \mathrm{NG}=$ neovascular glaucoma . $\mathrm{TD}=$ traction detachment. $\mathrm{OA}=$ optic atrophy.

Table 2 Course of 32 control cases in this study

\begin{tabular}{lccl}
\hline & Cases & Improved & Deteriorated \\
\hline Retreatment & 9 & 6 & 3 \\
Previous PRP & 12 & 10 & 2 \\
\hline
\end{tabular}

Table 3 Course of retreatment cases and those that had previous panretinal photocoagulation (PRP)

\begin{tabular}{llll}
\hline & \multicolumn{2}{l}{ Control cases } & \\
\cline { 2 - 4 } & Total & Type II & Type I \\
& 32 & 27 & 5 \\
\hline Spont. regression & 1 & 0 \\
Unchanged & 6 & 0 \\
Deteriorated & 20 & 5 \\
VH & 9 & 1 \\
TD & 6 & 2 \\
NG & 5 & 2 \\
OA & 0 & 0 \\
\hline
\end{tabular}

$\mathrm{VH}=$ vitreous haemorrhage. $\mathrm{NG}=$ neovascular glaucoma. $\mathrm{TD}=$ traction detachment. $\mathrm{OA}=$ optic atrophy.

Of 32 control cases (Table 3 ) five were of type I diabetes and 27 of type II or maturity onset diabetes. Only one maturity onset cases was observed to have regressed spontaneously, with quietening down of the neovascular activity and clearing of the vitreous blood. More gliosis was observed over the years, but that did not appear to alter significantly the visual function. Only six cases $(22 \%)$ of this type of diabetes remained almost unchanged, while 20 $(74 \%)$ deteriorated. Of the 20 eyes nine continued to bleed in the vitreous, six developed extensive traction detachment $(22 \%)$, and five ended up in neovascular glaucoma (18.5\%). All five eyes belonging to juvenile patients steadily deteriorated, with repeated attacks of vitreous haemorrhage in one eye, traction detachment in two, and neovascular glaucoma in two. 


\section{Discussion}

External diathermy appears to be the logical treatment of proliferative retinopathy when photocoagulation is impossible. It is particularly indicated for patients with PDR previously treated by photocoagulation who sustain an attack of vitreous haemorrhage and a flare-up of the fibrovascular activity, which would demand more retinal coagulations. This condition had quietened in 10 out of 12 such cases, astonishingly quickly. In addition further coagulation with the xenon arc or argon laser photocoagulators became possible after the rapid absorption of vitreous blood following diathermy coagulations (13 cases). Alternatively, further diathermy coagulation can still be done ( 9 cases).

Massive vitreous haemorrhage associated with PDR is rarely absorbed spontaneously and often recurs before enough absorption has taken place. This precludes the possibility of retinal photocoagulation and delays effective treatment. Such cases responded surprisingly quickly to diathermy treatment. In some cases after only 24 hours the absorption of the vitreous blood was remarkable. This was especially so with small fibrovascular formations.

Although the trend at present is towards earlier vitrectomy for vitreous haemorrhage, this procedure is never indicated for recent recurrent vitreous haemorrhage. This leaves us with only one possibility - namely external diathermy. I have not met with any serious complications of this treatment, nor was it apparent that such treatment adversely affected the course of PDR. It was of no use in certain cases. Only 13 out of 53 eyes treated continued to deteriorate, and only two out of these developed neovascular glaucoma, less than $4 \%$. Of 32 control cases 25 eyes $(78 \%)$ deteriorated, and seven of these developed neovascular glaucoma.

Serious complications have been reported when vitrectomy was performed on such cases- $49 \%$ in the group of cases treated by Tolentino and Freeman. ${ }^{6}$

No serious complications were encountered with our technique of transcleral choroidoretinal coagulation with local steroid treatment. Routine subconjunctival steroid and postoperative topical steroid were administered. Rarely systemic steroid therapy had to be instituted.

We have combined the buckling technique without drainage of subretinal fluid ${ }^{1}$ with external diathermy for PDR with traction detachment, and did paracentesis to control the tension, with good results. But we found that buckling and external diathermy with drainage were more effective in these cases (the results are not included in this study).

\section{PROGNOSTIC FACTORS}

A fairly good prognosis was associated with less florid PDR and less fibrovascular density. It was also better with less vitreous blood of less duration. Better visual prognosis was also associated with a good visual acuity before the attack of vitreous haemorrhage and with favourable ultrasonographic findings.

\section{CONCLUSION}

A controlled study of the role of diathermy treatment for diabetic vitreous haemorrhage and partial traction detachment has shown that a beneficial effect was observed in more than half of the cases. This line of treatment afforded a sound alternative to retinal photocoagulation and allowed the subsequent application of photocoagulation to a number of eyes. It also reduced the incidence of neovascular glaucoma considerably in the eyes that continued to deteriorate (two out of 53 cases, less than $4 \%$ ) after diathermy treatment compared with seven out of 25 eyes that deteriorated without treatment ( $22 \%$ of 32 cases).

\section{References}

1 Okun E, Fung WE. Therapy of diabetic retinal detachment. Symposium on retina and retinal surgery. Transactions of the New Orleans Academy of Ophthalmology. St Louis: Mosby, 1969: 319-27.

2 Wessing A, Bockenhoff I. Diathermy coagulation of diabetic proliferating retinopathy. Klin Monatsbl Augenheilkd 1971; 158: 212-20.

3 Siam A-L. Course, prognosis and management of proliferative diabetic retinopathy. Metab Pediatr Syst Ophthalmol 1981; 5: 109-10.

4 Schimek RA, Spencer WB. Cryopexy treatment of proliferative diabetic retinopathy. Retinal cryoablation in patients with severe vitreous haemorrhage. Arch Ophthalmol 1979; 97: 1276.

5 Michels RG. Vitreous surgery in proliferative diabetic retinopathy. XXIII Concilium ophthalmologicum Kyoto, 1978. Amsterdam: Excerpta Medica, 1978: 420.

6 Tolentino FL, Freeman HM. General aspects of closed vitrectomy through the pars plana. XXIII Concilium ophthalmologicum Kyoto, 1978. Amsterdam: Excerpta Medica 1978: 408.

Accepted for publication 6 June 1985. 